\title{
An analytical hierarchical model explaining the robustness and flaw- tolerance of the interlocking barb-barbule structure of bird feathers
}

\author{
Qiang Chen ${ }^{1(a)}$, Stanislav Gorb², Alexander Kovalev², Zhiyong Lii ${ }^{1,3}$ Nicola Pugno ${ }^{4,5,6}$ \\ ${ }^{1}$ Biomechanics Laboratory, School of Biological Science \& Medical Engineering, Southeast University Sipailou 2, \\ 210096 Nanjing, PRC \\ 2 Functional Morphology and Biomechanics, Kiel University - Am Botanischen Garten 1-9, D-24118 Kiel, Germany \\ ${ }^{3}$ School of Chemistry, Physics and Mechanical Engineering, Queensland University of Technology (QUT) Brisbane, \\ QLD 4001, Australia \\ ${ }^{4}$ Laboratory of Bio-Inspired \& Graphene Nanomechanics, Department of Civil, Environmental and Mechanical \\ Engineering, University of Trento - I-38123 Trento, Italy \\ ${ }^{5}$ Centre for Materials and Microsystems, Fondazione Bruno Kessler - Via Sommarive 18, I-38123 Povo (Trento), Italy \\ ${ }^{6}$ School of Engineering and Materials Science, Queen Mary University of London - Mile End Road E14NS, London, UK
}

\begin{abstract}
Feathers can fulfill their aerodynamic function only if the pennaceous vane forms an airfoil stabilized by robust interlocking between barbules. Thus, revealing the robustness of the interlocking mechanical behavior of the barbules is very important to understand the function and long-term resilience of bird feathers. This paper, basing on the small- and large-beam deflection solutions, presents a hierarchical mechanical model for deriving the critical delamination conditions of the interlocking barbules between two adjacent barbs in bird feathers. The results indicate a high robustness and flaw-tolerant design of the structure. This work contributes to the understanding of the mechanical behavior of the robust interlocking barb-barbule structure of the bird feather, and provides a basis for design of feather-inspired materials with robust interlocking mechanism, such as advanced bio-inspired micro-zipping devices.
\end{abstract}

\section{Introduction}

Birds' feathers are a complex evolutionary novelty characterized by hierarchical structural diversity. Regarding their structural morphogenesis, different researchers presented different models, from the developmental approach of molecular mechanisms [1], to the growth theory of feather [2], and to the competition between growth rate and feather quality [3]. These models have been analyzed in detail by following their functions, such as thermal insulation, water repellency, and mechanical protection [4]. One of their important functions, which are well-accepted, is that the feathers must possess the aerodynamic properties providing the birds' flight. This ability is achieved thanks to the rigid and flexible closed pennaceous vane, whose robustness or integrity are based on the elaborate interlocking of barbules (fig. 1(a)) [5]. Therefore, knowing the barbules' interlocking mechanics is very important to understand the function and robustness of the feathers. Recently, a mechanical experiment was performed to test the rupture and recovery behaviors of a swan (Cygnus olor ) feather in order to explore the zipping and unzipping behaviors in feathers [6]. It showed that the delamination strength (at rupture) of the interlocking barbules was dependent on the interaction between the bow- and hook-barbules, and the hooklets on the hook-barbules play an important role. In respect of hooklet mechanics, researchers have reported mechanical behaviors of single hooklets $[7,8]$, however, they are plants fruits for dispersing their seeds. Here, instead of studying the developmental morphology of feathers, we aimed at understanding the robustness of interlocking hook- and bow-barbule arrays in bird feathers, using a hierarchical analytical model (hook- and bowbarbules connected by microhook arrays). The deformations of the barbs and barbules were studied, 
and then coupled to develop a theoretical framework to predict critical delamination forces between the barbules. It is worth mentioning that the model does not include the exact hooklet geometry, and only considers the equivalent friction effect produced by the hooklets between the barbules as reasonably suggested by $[7,8]$.

\section{Theory}

According to the study on feather morphology [4], we first simplify the delaminated barbbarbule structure into a symmetrical hierarchical model, see fig. 1(b). The model involves deformations of barbs and barbules; here, we denote the barbs by superscript (1) and the barbules by superscript (2), respectively. Because of the symmetry about the global axis X (or OO'), the half structure of the $\mathrm{i}$-th pair of interlocking barbules $(1 \leq \mathrm{i} \leq \mathrm{k} \leq \mathrm{n}$, where $\mathrm{n}$ is the total number of pairs of hook- and bowbarbules, and $k$ is the number of pairs of interlocking hook- and bow-barbules, for fig. $1(b), n=9$ and $k=6)$ is analyzed, see fig. $1(\mathrm{c})$. In the analysis, three coordinate systems are introduced: one is global (X, Y) (fig. 1(b)), and the other two are local (xi, yi) and auxiliary (x i, y i) (fig. 1(c)). The auxiliary $(\mathrm{x} \mathrm{i}, \mathrm{y} \mathrm{i})$ is introduced for the transition transformation of the barbules from the local (xi, $\mathrm{yi})$ to the global $(X, Y)$. Before the analysis, we made the following two basic assumptions:

1) Due to the bending rigidity $D(2)$ of the barbules, which is much lower than $D(1)$ of the barbs, the deformation influence of the barbules on the barbs is assumed to be very weak, and thus is neglected; in contrast, the deformation influence of the barbs on the barbules is taken into account.

2) Sliding behavior between hook- and bow-barbules is assumed to be friction-dominated, which indicates the connecting barbules detach immediately when the angle coordinate $\beta i$ at point $B$ satisfies $\beta i-\Theta i=\psi>\psi f$ rict, where $\psi f$ rict is the friction angle and denotes a critical detaching state, and its tangent equals the ratio of the tangential to normal forces at the connecting point; if the ratio is greater than the tangent, sliding occurs.

Small deflection of barbs. According to the experimental observation in [6], we consider that the barb can be described assuming small deflection in the beam (here cantilever with one end fixed) theory; a set of dimensionless quantities is first defined as $K(1)=L(1) F / D(1), X=X / L(1), Y=Y / L(1), \Delta Y=$ $\Delta Y / L(1)$, where $F$ is the applied detaching force acting on the barb, $K(1)$ is the dimensionless detaching force, $\mathrm{L}(1)$ and $\mathrm{D}(1)$ are the length and bending rigidity of single barb, respectively, $(\mathrm{X}, \mathrm{Y})$ is coordinate of an arbitrary point on the barb, and correspondingly, $(X, Y)$ are its dimensionless coordinates, $\Delta \mathrm{Y}$ calculated by the classical Euler beam theory, is the deflection of the point in the $\mathrm{Y}$ direction. Then, for the upper barb in fig. 1(b), $Y$ is expressed as $Y=-I$ (2) $\sin \alpha 0 / L(1)-\Delta Y$, where $\alpha 0$ is an initial included angle made by the undeformed barbule and barb, and I (2) is the effective or deformed length (note: not the total length $L(2)$, fig. 1(b)) of the barbule. For the i-th locking barbules, the displacement $\Delta \mathrm{Yi}$ and rotation angle $\mathrm{Oi}$ of the cross-section at the joint coordinate $\mathrm{Xi}$ are expressed as [9]

$$
\left\{\begin{array}{l}
\Delta \bar{Y}_{i}=\frac{1}{6}\left(K^{(1)}\right)^{2} \bar{X}_{i}^{2}\left(3-\bar{X}_{i}\right), \\
\Theta_{i}=\frac{1}{2}\left(K^{(1)}\right)^{2} \bar{X}_{i}\left(2-\bar{X}_{i}\right) .
\end{array}\right.
$$

Large deflection of barbules. Similarly, according to the experimental observation in [6], there is an apparent rotation of barbules at the joint 0 , and thus, we consider that the barbules display large deflection of a beam, here a cantilever with one end rotationally constrained by an angular spring. Its deformation process is subdivided into two steps: according to the assumption (1), the barbules are influenced by the deformation of barbs, therefore, the first step is that it is translated by $\Delta \mathrm{Y}^{-} \mathrm{i}$ and rotates by $\Theta i$ caused by the barb with respect to the joint o, i.e., from the state IS to the MS state (fig. 1(c)): in this step, the barbule bears no force at the point A. The second step is that the barbule 
is deformed from the MS state to the FS state (fig. 1(c)), due to an equivalent interlocking force fi applied at the point $A$ which moves to the point $B$, moreover, under the interlocking force fi, the included angle made by barbs and barbules is changed from $\alpha 0$ into $\alpha 0+\alpha i$ (FS state in fig. 1(c)), where $\alpha i$ is the rotation angle of the $\mathrm{i}$-th barbule with respect to the joint $\mathrm{o}$. For the sake of expedience, we study the deformation process of the second step in the local system (xi, yi) (fig. 1(d)).

For the deformed portion $\mathrm{OB}$ in the barbules, the moment equilibrium with respect to a point $\mathrm{P}(\mathrm{xoB} \mathrm{i}$ , yoB i ) requires [10] $D(2) d \theta / d s=f i \cos \Theta i(l(2) \cos \alpha 0-\delta x i-x o B i)+f i \sin \Theta i(I(2) \sin \alpha 0+\delta y i-y o B$ $i$ ), where $D(2)$ is the bending rigidity of single barbule, $\delta x i$ and $\delta y i$ are the displacements of the point $B$ with respect to its original position $A$ in the xi and yi directions, respectively. Defining a new set of dimensionless quantities $\mathrm{K}(2) \mathrm{i}=\mathrm{I}(2) \mathrm{fi} / \mathrm{D}(2), \mathrm{s}=\mathrm{s} / \mathrm{I}(2), \delta \mathrm{xi}=\delta \mathrm{xi} / \mathrm{I}(2), \delta y \mathrm{i}=\delta \mathrm{yi} /(2), x \circ \mathrm{B} \mathrm{i}=\mathrm{xoB} \mathrm{i} / \mathrm{I}(2)$, yoB $\mathrm{i}=$ yoB $\mathrm{i} /(2)$, the above moment-equilibrium equation

$$
\begin{aligned}
\frac{\mathrm{d} \theta}{\mathrm{d} \bar{s}}= & \left(K_{i}^{(2)}\right)^{2} \cos \Theta_{i}\left(\cos \alpha_{0}-\bar{\delta}_{x_{i}}-\bar{x}_{i}^{o B}\right) \\
& +\left(K_{i}^{(2)}\right)^{2} \sin \Theta_{i}\left(\sin \alpha_{0}+\bar{\delta}_{y_{i}}-\bar{y}_{i}^{o B}\right) .
\end{aligned}
$$

Furthermore, deriving the curvature eq. (2) with respect to $\mathrm{s}$, and considering $\mathrm{dxoB} \mathrm{i} / \mathrm{ds}=\cos \theta$ and dyoB $\mathrm{i} / \mathrm{ds}=\sin \theta$, the equation is rearranged as

$$
\frac{\mathrm{d}^{2} \theta}{\mathrm{d} \bar{s}^{2}}=-\left(K_{i}^{(2)}\right)^{2} \cos \left(\theta-\Theta_{i}\right)
$$

Then, employing the boundary condition at the free end $B$, where the moment (or the curvature) is zero, i.e., $d \theta / d s \mid \theta=\beta i=0$, the curvature of the cantilever is calculated as

$$
\frac{\mathrm{d} \theta}{\mathrm{d} \bar{s}}=\sqrt{2} K_{i}^{(2)} \sqrt{\sin \left(\beta_{i}-\Theta_{i}\right)-\sin \left(\theta-\Theta_{i}\right)},
$$

where $\theta$ varies from $\alpha 0+\alpha i$ to $\beta \mathrm{i}$. According to the assumption (2), the condition $0<\beta i-\Theta i<\psi$ frict ensures the interlock of barbules. Integrating eq. (4) leads to the dimensionless curvilinear coordinate of $\mathrm{P}(\mathrm{xoB} \mathrm{i}$, yoB $\mathrm{i})$ :

$$
\begin{aligned}
\bar{s}(\theta)= & \frac{p_{i}}{K_{i}^{(2)}}\left[F\left(p_{i}, \frac{\theta-\Theta_{i}}{2}+\frac{\pi}{4}\right)\right. \\
& \left.-F\left(p_{i}, \frac{\alpha_{0}+\alpha_{i}-\Theta_{i}}{2}+\frac{\pi}{4}\right)\right],
\end{aligned}
$$

where $p 2 i=2 /[1+\sin (\beta i-\Theta i)]$, the function $F(p i, \xi)$ is the incomplete elliptical integral of the first kind, in which $\xi$ is a general amplitude. Considering the inextensibility of the cantilever ${ }^{-} s(\beta i)=1$, we find that

$$
\begin{aligned}
K_{i}^{(2)}= & p_{i}\left[F\left(p_{i}, \frac{\beta_{i}-\Theta_{i}}{2}+\frac{\pi}{4}\right)\right. \\
& \left.-F\left(p_{i}, \frac{\alpha_{0}+\alpha_{i}-\Theta_{i}}{2}+\frac{\pi}{4}\right)\right],
\end{aligned}
$$

based on $d^{-} x o B i / d^{-} s=\cos \theta, d^{-} y o B i / d^{-} s=\sin \theta$, and eq. (4), the dimensionless Cartesian coordinates of the point $\mathrm{P}\left({ }^{-} \mathrm{xoB} i, \mathrm{y}^{-} \mathrm{oB} \mathrm{i}\right)$ can be computed as 


$$
\left\{\begin{array}{l}
\bar{x}_{i}^{o B}(\theta)=\int_{\alpha_{0}+\alpha_{i}}^{\theta} \cos \theta \mathrm{d} \bar{s}=\frac{1}{\sqrt{2} K_{i}^{(2)}} \int_{\alpha_{0}+\alpha_{i}}^{\theta} \frac{\cos \left(\theta-\Theta_{i}+\Theta_{i}\right)}{\sqrt{\sin \left(\beta_{i}-\Theta_{i}\right)-\sin \left(\theta-\Theta_{i}\right)}} \mathrm{d} \theta=\frac{1}{\sqrt{2} K_{i}^{(2)}}\left(A(\theta) \cos \Theta_{i}-B(\theta) \sin \Theta_{i}\right), \\
\bar{y}_{i}^{o B}(\theta)=\int_{\alpha_{0}+\alpha_{i}}^{\theta} \sin \theta \mathrm{d} \bar{s}=\frac{1}{\sqrt{2} K_{i}^{(2)}} \int_{\alpha_{0}+\alpha_{i}}^{\theta} \frac{\sin \left(\theta-\Theta_{i}+\Theta_{i}\right)}{\sqrt{\sin \left(\beta_{i}-\Theta_{i}\right)-\sin \left(\theta-\Theta_{i}\right)}} \mathrm{d} \theta=\frac{1}{\sqrt{2} K_{i}^{(2)}}\left(A(\theta) \sin \Theta_{i}+B(\theta) \cos \Theta_{i}\right),
\end{array}\right.
$$

where

$$
\left\{\begin{aligned}
A(\theta)= & \int_{\alpha_{0}+\alpha_{i}}^{\theta} \frac{\cos \left(\theta-\Theta_{i}\right)}{\sqrt{\sin \left(\beta_{i}-\Theta_{i}\right)-\sin \left(\theta-\Theta_{i}\right)}} \mathrm{d} \theta= \\
& 2\left(\sqrt{\sin \left(\beta_{i}-\Theta_{i}\right)-\sin \left(\alpha_{0}+\alpha_{i}-\Theta_{i}\right)}-\sqrt{\sin \left(\beta_{i}-\Theta_{i}\right)-\sin \left(\theta-\Theta_{i}\right)}\right), \\
B(\theta)= & \int_{\alpha_{0}+\alpha_{i}}^{\theta} \frac{\sin \left(\theta-\Theta_{i}\right)}{\sqrt{\sin \left(\beta_{i}-\Theta_{i}\right)-\sin \left(\theta-\Theta_{i}\right)}} \mathrm{d} \theta= \\
& \sqrt{2} p_{i}\left[\sin \left(\beta_{i}-\Theta_{i}\right)\left(F\left(p_{i}, \frac{\theta-\Theta_{i}}{2}+\frac{\pi}{4}\right)-F\left(p_{i}, \frac{\alpha_{0}+\alpha_{i}-\Theta_{i}}{2}+\frac{\pi}{4}\right)\right)\right. \\
& \left.-\frac{2}{p_{i}^{2}}\left(E\left(p_{i}, \frac{\theta-\Theta_{i}}{2}+\frac{\pi}{4}\right)-E\left(p_{i}, \frac{\alpha_{0}+\alpha_{i}-\Theta_{i}}{2}+\frac{\pi}{4}\right)\right)\right],
\end{aligned}\right.
$$

in which the function $\mathrm{E}(\mathrm{pi}, \xi)$ is the incomplete elliptical integral of the second kind. Then, transforming the coordinates of the barbules from the local coordinate system (xi, yi) to the global one $(X, Y)$, the corresponding global coordinates of the barbules are obtained as

$$
\begin{aligned}
\left(\begin{array}{l}
\bar{X}_{i}^{o B}(\theta) \\
\bar{Y}_{i}^{o B}(\theta)
\end{array}\right)= & \left(\frac{L^{(1)}}{l^{(2)}}\right)\left(\begin{array}{c}
\bar{X}_{i} \\
\bar{Y}_{i}
\end{array}\right) \\
& +\left(\begin{array}{cc}
\cos \Theta_{i} & \sin \Theta_{i} \\
-\sin \Theta_{i} \cos \Theta_{i}
\end{array}\right)\left(\begin{array}{l}
\bar{x}_{i}^{o B}(\theta) \\
\bar{y}_{i}^{o B}(\theta)
\end{array}\right)= \\
& \left(\frac{L^{(1)}}{l^{(2)}}\right)\left(\begin{array}{c}
\bar{X}_{i} \\
\bar{Y}_{i}
\end{array}\right)+\frac{1}{\sqrt{2} K_{i}^{(2)}}\left(\begin{array}{l}
A(\theta) \\
B(\theta)
\end{array}\right),
\end{aligned}
$$

where $\mathrm{XoB} i(\theta)=\operatorname{XoB} i(\theta) /(2), \operatorname{YoB} i(\theta)=\mathrm{YoB} i(\theta) /(2)$, and $(\mathrm{Xi}, \mathrm{Yi})$ is the dimensionless coordinate of the $\mathrm{i}$-th joint $\mathrm{o}$. In particular, the coordinates $(\mathrm{XoB} \mathrm{i}(\beta \mathrm{i})$, YoB $\mathrm{i}(\beta \mathrm{i}))$ of the point $\mathrm{B}$ at the $\mathrm{i}$-th barbule are obtained from eq. (8). In the global system, because the ordinate of the point B equals zero, i.e., YoB i $(\beta i)=0$, we have

$$
K_{i}^{(2)}=\frac{B\left(\beta_{i}\right)}{\sqrt{2}\left[\sin \alpha_{0}+\Delta \bar{Y}_{i}\left(L^{(1)} / l^{(2)}\right)\right]} .
$$

Again, considering moment equilibrium of the cantilever but with respect to the joint $\mathrm{o}$, a new equation emerges:

$$
\begin{aligned}
\lambda \alpha_{i}= & f_{i} \cos \Theta_{i} x_{i}^{o B}\left(\beta_{i}\right)+f_{i} \sin \Theta_{i} y_{i}^{o B}\left(\beta_{i}\right)= \\
& \frac{f_{i} l^{(2)}}{\sqrt{2} K_{i}^{(2)}} A_{i}\left(\beta_{i}\right),
\end{aligned}
$$

where $\lambda$ is the stiffness of angular spring (which has the physical unit $(\mathrm{N} \cdot \mathrm{m} / \mathrm{rad})$ ). Rearrangement of the above equation leads to

$$
K_{i}^{(2)}=\frac{\bar{\lambda} \alpha_{i}}{\sqrt{2\left[\sin \left(\beta_{i}-\Theta_{i}\right)-\sin \left(\alpha_{0}+\alpha_{i}-\Theta_{i}\right)\right]}},
$$

where $\lambda=\lambda(2) / D(2)$ is a new dimensionless parameter, which represents the materials parameters in the barbbarbule system, i.e., the relative relationship between the rotation and bending abilities of the barbules. Thus, substituting eq. (11) into eqs. (6) and (9), an equation system with two unknown 
parameters, ai and $\beta \mathrm{i}$, could be solved numerically. With the solution of $\alpha \mathrm{i}$ and $\beta \mathrm{i}$, the dimensionless force $\mathrm{K}(2) \mathrm{i}$ in each barbule can be calculated.

Again, invoking $\mathrm{YoB} i(\beta i)=0$, the coordinates of the straight portion $\mathrm{BC}$ part satisfy

$$
\bar{Y}_{i}^{B C}=\tan \left(\beta_{i}-\Theta_{i}\right)\left[\bar{X}_{i}^{B C}-\bar{X}_{i}^{o B}\left(\beta_{i}\right)\right],
$$

where $Y B C i=Y B C i / /(2), X B C i=X B C i / /(2)$ is from $X o B i(\beta i)$ to $X o B i(\beta i)+(L(2) / /(2)-1) \cos (\beta i-$ అi).

The above equations from (2) to (12) are derived for the interlocking barbules. As for the detached barbules $(k+1 \leq i \leq n)$, the coordinates of the barbules satisfy

$$
\bar{Y}_{i}^{o C}=\tan \left(\alpha_{0}-\Theta_{i}\right)\left[\bar{X}_{i}^{o C}-\bar{X}_{i}\left(\frac{L^{(1)}}{l^{(2)}}\right)\right]+\bar{Y}_{i}\left(\frac{L^{(1)}}{l(2)}\right),
$$

where $\mathrm{YoC} i=\mathrm{Y}$ oC $\mathrm{i} /((2), \mathrm{XoC} \mathrm{i}=\mathrm{XoC} \mathrm{i} / /(2)$ which varies from $\mathrm{Xi}(\mathrm{L}(1) /((2))$ to $\mathrm{Xi}(\mathrm{L}(1) /((2))+$ $(L(2) /(2)) \cos (\alpha 0-\Theta i)$, and $(X i, Y i)$ is the same as that in eq. (8).

\section{Parametric analysis and discussions}

As an example, the eight critical states in the delaminating process shown in fig. 1(b) are treated; we selected an initial included angle $\alpha 0=30^{\circ}$, according to the statistic data of the barbules angle in the literature [11]. The dimensionless rotation stiffness $\lambda^{-}$of the barbules and the friction angle $\psi f$ rict are assigned to be 1.0 and $45^{\circ}$, respectively, and length ratios used in the model are set to be $\mathrm{L}(2) /(2)=1.2$ and $\mathrm{L}(1) / /(2)=10$.

In each critical state, the k-th pair of barbules detaches, and the connecting dimensionless forces $\mathrm{K}(2) \mathrm{i}$, the rotation angles ai and $\beta \mathrm{i}$ in the $\mathrm{i}$-th $(\mathrm{i} \leq \mathrm{k})$ interlocking barbules are plotted in fig. 2 . We can see that when $i=1$ and $K(2) i=0$, $\alpha i=0$ and $\beta i=30$ 。 for the fixed first barbule; whereas for $2 \leq i$ $\leq \mathrm{k}$, the distribution of the dimensionless forces $\mathrm{K}(2) \mathrm{i}$ in the interlocking barbules is linear. The force on the same barbule decreases as the number $k$ of interlocking barbules increases. For instance, the force on the 2nd barbule decreases as indicated by the dashed arrow in fig. 2(a), when $\mathrm{k}$ increases from 2 to 9 . This means that the detaching force acting on the 2nd barbule, which determines the ultimate structural robustness, is mitigated by the presence of interlocking barbules behind it. The rotation angles ai and $\beta i$ are nonlinearly distributed (fig. 2(b)). Also, the increasing number of interlocking barbules decreases the two rotation angles and thus the structural failure probability. Accordingly, the structure consisting of multiple barbules may progressively but effectively absorb energy before the complete structural failure, and thus enhance its robustness, and this may be a reason why there are many instead of few barbules on a barb [6].

Besides, the critical detaching force $K(2) k$,crit of the barbule in each critical state and its corresponding critical applied forces $\mathrm{K}(1) \mathrm{k}$,crit of the barb are plotted in fig. 3(a), and the deformed profiles of the critical states are plotted in fig. 3(b). In fig. 3(a), both critical forces decrease as the number $\mathrm{k}$ of interlocking barbules increases; in particular, $\mathrm{K}(1) \mathrm{k}$,crit decreases sharply when $\mathrm{k}$ changes from 2 to 3 . This is because the barbules closer to the fixed ends of barbs are severer restrained, compared to the barbules far from the ends, and a larger force is needed to detach the barbules situated closer to the fixed end. Surprisingly, the local force on individual barbules with increased number of detached barbules ( $k$ from 9 to 2 ) is increased only 1.3 times, whereas the critical separation force increases 7 times. In this regard, the structural hierarchy and large rigidity difference seem to propose a strong gradient failure of barbs and a weak one of barbules in the interlocked architecture, which is crucial for reaching the high resistance to quasistatic (fatigue) and 
dynamical loadings during the flight in birds' pennaceous feathers. Corresponding to fig. 3(a), the profiles plotted in fig. 3(b) indicate that detaching the $k$-th $(k \geq 3)$ barbules is easier because of the weak de- flection differences between these barbules, compared to $k=2$ (the thick blue line).

In all, according to the existence of a large number of barbules, fig. 2, and to the different gradient failures of barbs and barbules in the static delamination, fig. 3, the pennaceous vanes are not seriously disclosed when birds are flying, despite the absence of some barbules (see fig. 3(a), when the number of absent barbules $\mathrm{FN}=1,2,3$, the delamination is not strongly influenced). This suggests a flaw-tolerant and robust behavior of this natural and hierarchical design. However, we must admit that due to the neglected influence of barbules on barbs, the detachment of barbules is forced not to cause the instability in the progressive delamination, even if a dynamic instable process could take place.

\section{Conclusions}

Revealing the detaching behavior of the barb-barbule structure in birds' feather is very helpful to understand their aerodynamic function. This paper employed beam theory to develop a hierarchical mechanical model to compute the static delamination strength of the barb-barbule system in the bird feather. The model results indicate a high robustness and thus flaw-tolerance of the natural design, and suggest a new bio-inspired strategy in the design of robust interlocking mechanism, such as bioinspired zipping devices.

\section{Acknowledgements}

This work is partially supported by the National Natural Science Foundation of China (NSFC) (No. 31300780, 11272091, 11422222, 31470043), the Fundamental Research Funds for the Central Universities (No. 2242016R30014), and ARC (FT140101152). NMP is supported by the European Research Council (ERC StG Ideas 2011 BIHSNAM no. 279985, ERC PoC 2015 SILKENE No. 693670) and by the European Commission under the Graphene Flagship (WP14 "Polymer Composites", No. 696656).

\section{References}

[1] Prum R. O., J. Exp. Zool., 285 (1999) 291.

[2] Yu M., Wu P., Widelitz R. B. and Chuong C.-M., Nature, 420 (2002) 308.

[3] De La Hera I., Perez-Tris J. and Telleria J. L., Biol. J. Linn. Soc., 97 (2009) 98.

[4] Prum R. O. and Williamson S., J. Exp. Zool., 291 (2001) 30.

[5] Miller S. A. and Harley J. P., Zoology, 5th edition (Mcraw-Hill, New York) 2001, p. 321.

[6] Kovalev A., Filippov A. E. and Gorb S. N., J. R. Soc. Interface, 11 (2014) 20130988.

[7] Pugno N. M., Appl. Phys. Lett., 90 (2007) 121918.

[8] Chen Q., Gorb S., Gorb E. and Pugno N. M., J. R. Soc. Interface, 10 (2013) 20120913.

[9] Young W. C. and Budynas R. G., Roark's Formulas for Stress and Strain, 7th edition (McGrawHill, New York) 2001, p. 189.

[10] Bisshopp K. E. and Drucker D. C., Q. Appl. Math., 3 (1945) 272. 
[11] Bachmann T., Klan S., Baumgartner W., Klaas M., " Schroder W. " and Wagner H., Front. Zool., 4 (2007) 23.

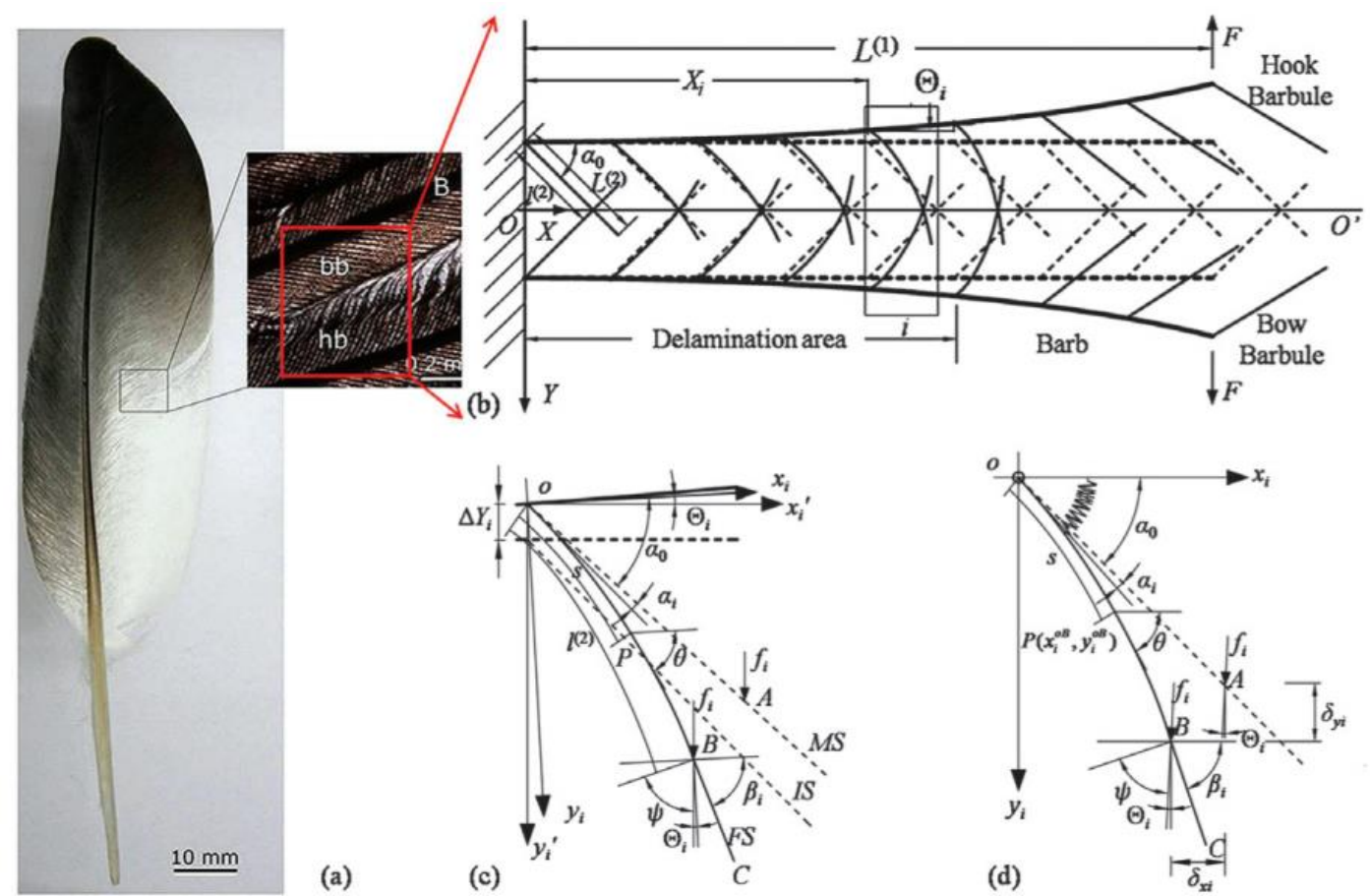

Fig. 1: (Colour online) A bird feather and symmetrical delamination of the hierarchical barb-barbule model (barbs in thick lines and barbules in thin ones) for feathers. (a) A third primary pennaceous feather of Columba oenas and a light microscopy image of the vane, where a barb (B), hook barbules (hb) and bow barbules (bb) are indicated. (b) Symmetrical delamination model of the structure, before (dashed lines) and after delamination (solid lines). (c) A basic large-displacement cantilever-beam model and force analysis for barbules in the global system $(X, Y)$. (d) Force analysis on the $i$-th barbules in the local system $(x, y)$.
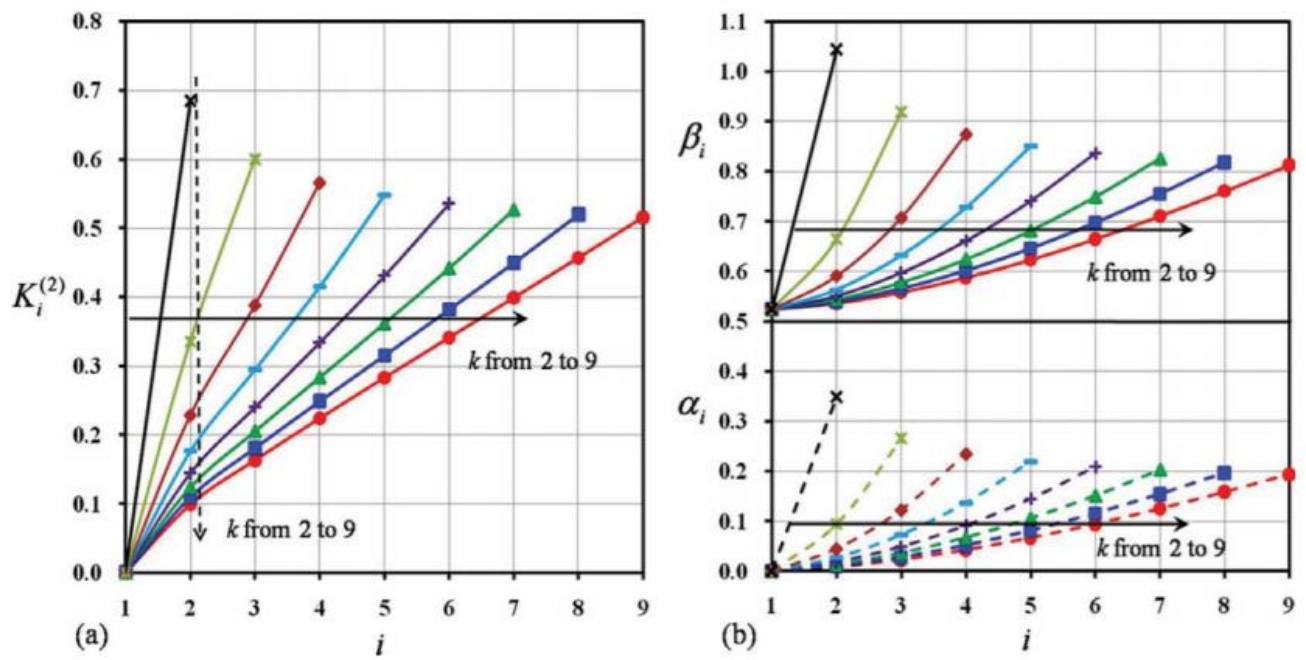

Fig. 2: (Colour online) Dimensionless forces and rotation angles of the barbules in eight critical states. (a) Interlocking forces of the barbules. (b) Rotation angles of both ends of the cantilever barbule model. 

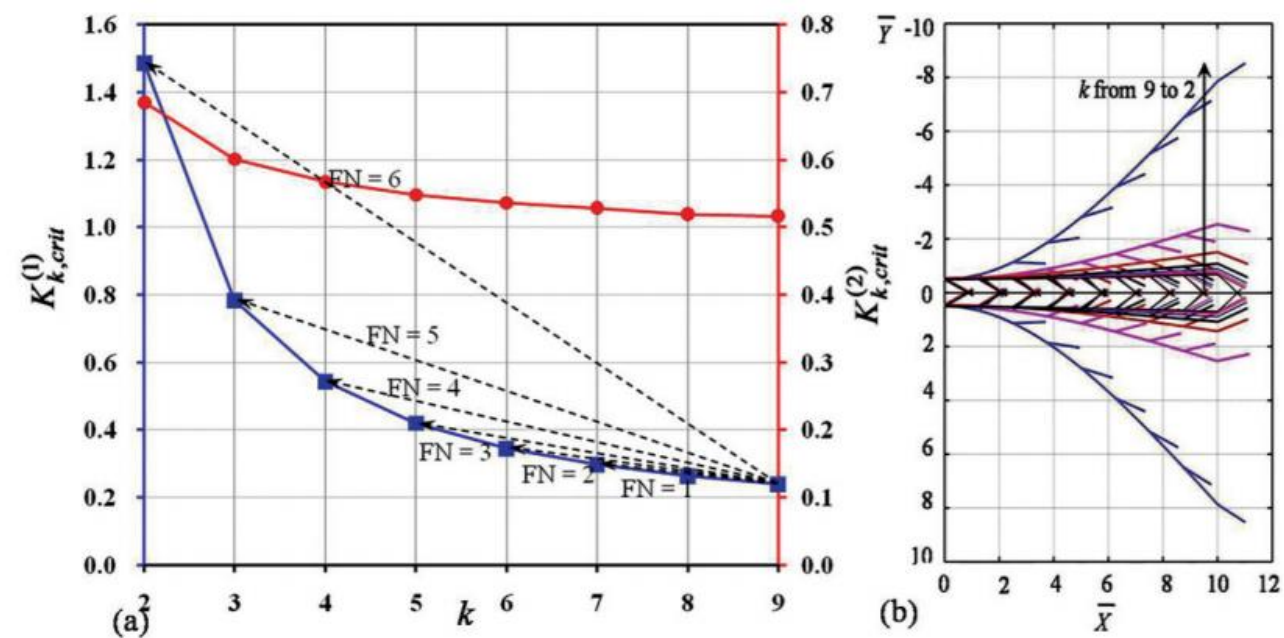

Fig. 3: (Colour online) Critical dimensionless forces and profiles. (a) Critical forces of the $k$-th detached barbule (solid circles) $K_{k, \text { crit }}^{(2)}$, and the corresponding critical applied force on the barb (solid squares) $K_{k, c r i t}^{(1)}$. (b) The deformed profiles of eight critical states. Note: FN denotes the number of absent barbules. 